\title{
ESTUDOS DA INFLUÊNCIA DA BASICIDADE E DO TEOR DE FeO DO SINTER NA ZONA DE AMOLECIMENTO E FUSÃO DO ALTO FORNO*
}

\author{
Aline Aguiar Lopes ${ }^{1}$ \\ Arnaldo Ledig Aguiar Silva ${ }^{2}$ \\ Fernando Tadeu Pereira de Medeiros ${ }^{3}$ \\ José Adilson de Castro ${ }^{4}$ \\ Marcelo Alves de Carvalho 5 \\ Sebastião Jorge Xavier Noblat ${ }^{6}$
}

\section{Resumo}

A piora na qualidade dos minérios de ferro tem levado as empresas a adaptarem seus processos a novas condições de qualidade das matérias-primas, surgindo assim a necessidade de desenvolver estudos que auxiliem as tomadas de decisão. Esse trabalho avaliou a influência da qualidade do sínter e da composição da carga metálica na zona coesiva do alto-forno através dos parâmetros de amolecimento e fusão. A importância de se obter informações sobre a zona coesiva se deve principalmente ao fato de nessa região ocorrer a maior parte da perda de carga no interior do alto-forno. Cinco amostras de sínter de qualidades químicas distintas, produzidas nas sinterizações da CSN (Companhia Siderúrgica Nacional), foram selecionadas e caracterizadas para esse estudo. Os ensaios de Amolecimento e Fusão foram realizados no Centro de Tecnologia de Ferrosos da Vale através de um acordo de cooperação técnica entre as empresas. Os resultados obtidos mostraram que as técnicas utilizadas são adequadas para avaliação das propriedades em altas temperaturas, fornecendo dados importantes para a interpretação do comportamento da carga metálica no interior do alto-forno, como espessura e posicionamento da zona coesiva, permeabilidade e redutibilidade da carga, indicando assim, a composição da carga metálica de melhor desempenho para o alto-forno.

Palavras-chave: Zona coesiva; Carga metálica; Amolecimento \& Fusão; Alto-forno.

\section{STUDY ABOUT INFLUENCE OF THE SINTER'S BASICITY AND FEO CONTENT IN SOFTENING AND MELTING AREA OF THE BLAST FURNACE}

\section{Abstract}

The worsening of the iron ore has driven many companies to adapt their process to new raw material quality parameters, hence the need to develop new studies that helps in the process of decision making. This article's aim was to evaluate the influence of the sinter quality at the cohesive zone of the blast furnace through the ferrous burden softening e melting parameters. The importance of obtaining such informations about the cohesive zone is due, mainly, to the fact that most of the pressure drop occurs inside this region of the blast furnace. Five samples of sinter with different quimical analisys, produced at C.S.N's (Companhia Siderúrgica Nacional) sinter plant, where selected and characterized for this study. The softening and melting analysis where conducted at the C.T.F. (Centro de Tecnologia de Ferrosos)_due to a technical cooperation agreement between C.S.N. and Vale. The achieved results proves that the technics used on this research are suitable to evaluate the properties under high temperatures, providing important data to interpret how the metal burden behaves inside the blast furnace, such as thickness and the positioning, the burden's permeability and reducibility, indicating the metal burden composition that optimizes the blast furnace's performance.

Keywords: Cohesive zone; Ferrous burden; Softening \& Melting; Blast furnace.

1 Engenharia Metalúrgica, Mestrando no PPGEM - Uff, Engenheira de Desenvolvimento Sênior, Gerência de Processos Metalúrgicos, Companhia Siderúrgica Nacional (CSN), Volta Redonda, RJ, Brasil.

2 Engenharia Metalúrgica, Coordenador de Projetos Especiais, Gerência de Processos Metalúrgicos, Companhia Siderúrgica Nacional, Volta Redonda, RJ, Brasil.

3 Prof. Dr, Membro ABMM, Escola de Engenharia Industrial Metalurgia de Volta Redonda (EEIMVR), Universidade Federal Fluminense (UFF), Volta Redonda, RJ, Brasil.

4 Prof. Dr, Membro ABMM, EEIMVR, UFF, Volta Redonda, RJ, Brasil.

5 Eng. Metalúrgica, Gerente de Projetos de Infraestrutura, CSN, Volta Redonda, RJ, Brasil.

6 Eng. Met., Coord. de Tec. Met., Gerência de Processos Metalúrgicos, CSN, Volta Redonda, RJ, Brasil. 


\section{INTRODUÇÃO}

A busca contínua das empresas por melhor desempenho com menor custo impulsiona o desenvolvimento de novos estudos avançando em áreas antes menos exploradas. É sabido que a Zona Coesiva é a região em que ocorre a maior perda de carga no interior do alto-forno, o que implica em menor permeabilidade. Além disso, a espessura e o posicionamento da zona coesiva afetam o escoamento gasoso e consequentemente, a produtividade do alto-forno. $O$ presente trabalho tem o objetivo de avaliar a influência da qualidade do sínter na zona coesiva do alto-forno, através dos parâmetros de amolecimento e fusão da carga metálica, visto que este material compõem o maior percentual da carga utilizada nos altos-fornos e ainda, avaliar diferentes composições de carga metálica para uma mesma qualidade química de seus componentes.

Um método experimental para avaliação da carga metálica nessa região, conhecido como Ensaio de Amolecimento e Fusão (A\&F), avalia o comportamento da carga metálica em altas temperaturas, desde os estágios iniciais de aquecimento e redução até sua fusão completa, possibilitando assim, a determinação da espessura e altura da zona coesiva, além de avaliar a permeabilidade e redutibilidade da carga.

Alguns fatores conhecidos na literatura influenciam as características da carga metálica e suas propriedades a altas temperaturas, tais como basicidade, teor de $\mathrm{FeO}$, teor de $\mathrm{MgO}$, teor de $\mathrm{Al}_{2} \mathrm{O}_{3}$, entre outros [1] [2]. $\mathrm{O}$ foco desse estudo foi a basicidade e o teor de $\mathrm{FeO}$. Espera-se que sínter de Basicidade baixa possua temperatura de amolecimento mais baixa, porque tende a formar Faialita, além de piorar a redutibilidade (menor grau de pré-redução). Com isso, formam fase líquida mais cedo provocando gotejamento prematuro. Já o sínter de Basicidade alta, melhora as propriedades de amolecimento e fusão devido ao efeito do grau de redução obtido no início da zona coesiva. Tende a formar Silicatos de Cálcio de alto ponto de fusão. Menores teores de $\mathrm{FeO}$, conseguidos pela menor dosagem de combustível na mistura de sinterização, formam menos Faialita e melhoram a redutibilidade do Sínter.

Baseado nesses fatores teóricos, foram definidas as faixas de qualidade de sínter a serem estudadas, variando os valores de basicidade e teor de $\mathrm{FeO}$. As amostras foram produzidos na planta de sinterização da CSN. Com essas amostras foram realizados os ensaios de Amolecimento e Fusão, além da caracterização química.

\section{MATERIAIS E MÉTODOS}

\subsection{MATERIAIS}

Foram selecionadas uma amostra típica de pelota e uma de minério de ferro granulado para realização de todos os ensaios de A\&F, variando assim, somente a qualidade do sínter na composição da carga metálica. Cinco tipos de sínter de diferentes composições químicas foram selecionados e caracterizados, tendo como referência os teores de $\mathrm{FeO}$ e Basicidade (B2), sendo eles:

- Sínter base: B2 de 1,80 e $\mathrm{FeO}$ de 7,0\%.

- Sínter de alta basicidade: B2 de 2,0 e FeO de 7,0\%.

- Sínter de baixa basicidade: B2 de 1,60 e FeO de 7,0\%.

- Sinter de alto teor de FeO: B2 de 1,80 e FeO de 9,0\%.

- Sinter de baixo teor de FeO: B2 de 1,80 e FeO de 5,0\%. 


\subsection{MÉTODOS:}

\subsubsection{ANÁLISE QUÍMICA}

A análise química das amostras foi realizada por Espectrometria de Fluorescência de Raios-X (FRX) e os teores de $\mathrm{FeO}, \mathrm{Na}_{2} \mathrm{O}$ e $\mathrm{K}_{2} \mathrm{O}$ foram determinados por via úmida devido a maior confiabilidade dos resultados. Os ensaios foram realizados nos laboratórios da CSN, seguindo seus procedimentos internos.

\subsubsection{ENSAIOS DE AMOLECIMENTO E FUSÃO}

Os ensaios de Amolecimento e Fusão sob Carga foram realizados no CTF (Centro de Tecnologia de Ferrosos) da Vale seguindo metodologia própria, já que esse ensaio não é padronizado por nenhuma instituição como ISO ou ABNT. Foram selecionadas as seguintes cargas metálicas para os Leitos de Fusão:

- Carga metálica composta de sinter, minério granulado e pelota nas proporções típicas do Alto-forno 3 da CSN. Para essa composição de carga metálica foram realizados cinco ensaios de A\&F: Caso Base (qualidade típica do sínter), Análise II (com sinter de baixa Basicidade), Análise III (com sínter de alta Basicidade), Análise IV (com sínter de alto teor de FeO), Análise V (com sínter de baixo teor de $\mathrm{FeO}$ ).

- Carga metálica sem pelota no Leito de Fusão utilizando o sínter de qualidade típica, denominado Análise I.

- Carga metálica sem minério granulado no Leito de Fusão utilizando o sínter de qualidade típica, denominado Análise VI.

O procedimento do ensaio de A\&F conforme descrito por Castro, Pimenta e Carneiro [3] consiste no carregamento da amostra de carga metálica (individual ou mista) entre duas camadas de coque padrão de $20 \mathrm{~mm}$ cada, em um cadinho de grafite. O peso de cada material a ser carregado no cadinho é calculado para que a carga metálica em estudo atinja $70 \mathrm{~mm}$ de altura.

Após o carregamento com a amostra, o cadinho é posicionado na região central do forno superior. A amostra é aquecida até $800^{\circ} \mathrm{C}$ em uma atmosfera de nitrogênio sob uma taxa de aquecimento de $10^{\circ} \mathrm{C} /$ minuto. A partir daí uma pressão de $98 \mathrm{kPa}$ é aplicada na amostra e inicia-se o fluxo do gás redutor. Ao atingir $1000^{\circ} \mathrm{C}$ a taxa de aquecimento do forno superior é alterada para $5^{\circ} \mathrm{C} /$ minuto e o aquecimento prossegue até atingir $1600^{\circ} \mathrm{C}$ e até que ocorra o gotejamento do material. $\mathrm{O}$ aquecimento do forno inferior prossegue a $10^{\circ} \mathrm{C} /$ minuto até $1700^{\circ} \mathrm{C}$. A partir de $800^{\circ} \mathrm{C} \mathrm{em}$ diante o gás de saída é analisado continuamente permitindo a determinação do grau de redução da amostra. O deslocamento do pistão é monitorado, registrando a redução de volume devido ao amolecimento e fusão da amostra. O material fundido ao gotejar é coletado em amostradores localizados dentro da mesa giratória na região inferior do equipamento, cujo giro é iniciado automaticamente com a detecção do início do gotejamento por um sensor. A Figura 1 mostra os detalhes do equipamento.

Durante o ensaio de A\&F o sistema de aquisição de dados coleta as temperaturas do forno inferior, do forno superior e da amostra, temperatura de gotejamento, ocorrência de gotejamento, a queda de pressão na amostra, vazão de entrada do gás redutor e a composição dos gases de saída $\left(\mathrm{CO}, \mathrm{CO}_{2}\right.$ e $\left.\mathrm{H}_{2}\right)$. 


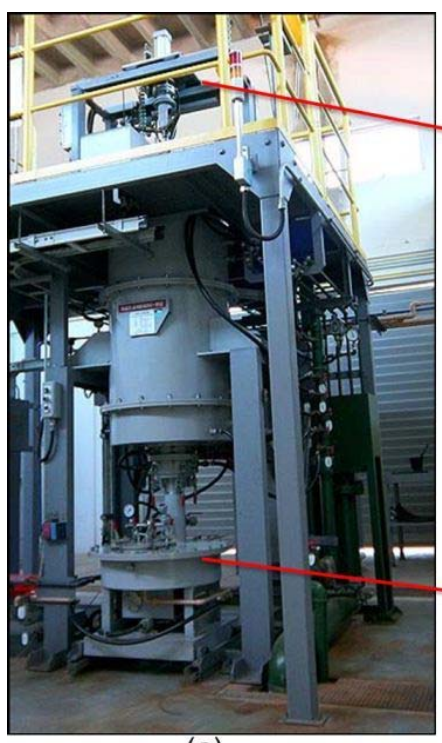

(a)

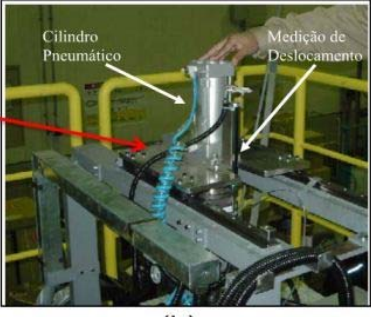

(b)

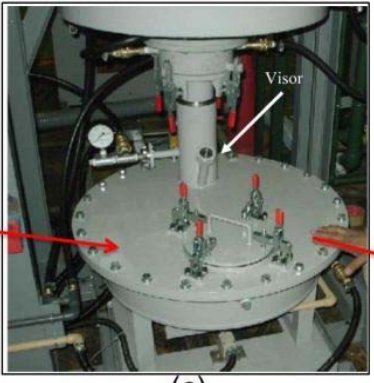

(c)

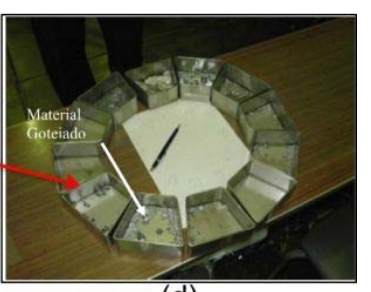

(d)

Figura 1: Detalhes do forno de A\&F. (a) Visão geral do forno. (b) Detalhe do aparato para aplicação e medição de pressão na carga. (c) detalhe da mesa giratória de coleta de amostras. (d) Recipientes para coleta das amostras. Adaptado de [1] [4].

Os principais parâmetros medidos no ensaio de Amolecimento e Fusão, são [5]:

- TS 200: temperatura correspondente ao início da zona coesiva.

- TE 200: temperatura correspondente ao final da zona coesiva.

- TD(“dripping"): Temperatura de gotejamento.

- $\quad \Delta \mathrm{T}$ : TE - TS; Corresponde ao aumento de temperatura experimentado pela carga metálica na região de queda de pressão mais significativa.

- Valor S: calculado pela área abaixo da curva de queda de pressão. É um indicador da permeabilidade global do leito durante o ensaio.

- $\Delta$ Pmax: Queda máxima de pressão.

- RS, R50, R1000, R1100 e R1200: correspondem ao grau de redução instantâneo nas temperaturas TS, TSh50, $1000^{\circ} \mathrm{C}, 1100^{\circ} \mathrm{C}$ e $1200^{\circ} \mathrm{C}$.

\section{RESULTADOS E DISCUSSÃO}

A Tabela 1 mostra o resultado da análise química das amostras utilizadas nos ensaios.

Tabela 1. Análise química

\begin{tabular}{|c|c|c|c|c|c|c|c|c|c|c|c|c|c|}
\hline Amostra & $\mathrm{FeT}$ & $\mathrm{FeO}$ & $\mathrm{MgO}$ & $\mathrm{Al} 2 \mathrm{O} 3$ & $\mathrm{SiO} 2$ & $\mathrm{CaO}$ & $\mathrm{P}$ & $\mathrm{Mn}$ & $\mathrm{B} 2$ & Vol. Escória & $\mathrm{Na} 2 \mathrm{O}$ & $\mathrm{K} 2 \mathrm{O}$ \\
\hline Sinter Base & 55,69 & 7,34 & 1,68 & 1,14 & 6,18 & 11,2 & 0,055 & 0,44 & 1,81 & 17,38 & 0,043 & 0,031 \\
\hline Sinter B2 baixa & 55,28 & 7,19 & 1,47 & 1,33 & 6,86 & 10,96 & 0,059 & 0,54 & 1,6 & 17,82 & 0,049 & 0,039 \\
\hline Sinter B2 alta & 55,1 & 6,54 & 1,67 & 0,89 & 5,96 & 12,47 & 0,047 & 0,57 & 2,09 & 18,43 & 0,028 & 0,044 \\
\hline Sinter FeO baixo & 55,13 & 4,24 & 1,7 & 1,11 & 6,32 & 11,44 & 0,053 & 0,52 & 1,81 & 17,76 & 0,044 & 0,025 \\
\hline Sinter FeO alto & 54,82 & 9,66 & 1,74 & 1,3 & 6,26 & 11,51 & 0,048 & 0,57 & 1,84 & 17,77 & 0,044 & 0,028 \\
\hline $\begin{array}{c}\text { Pelota } \\
\text { Minério } \\
\text { granulado }\end{array}$ & 64,39 & 0 & 0 & 0,95 & 3,96 & 2,85 & 0,043 & 0,06 & 0,72 & 6,81 & 0 & 0 \\
\hline
\end{tabular}

Os ensaios de Amolecimento e Fusão foram realizados conforme procedimento citado no item 2.2.2. A Figura 2 mostra a preparação do cadinho com as amostras e a Figura 3 mostra o momento de início de gotejamento de material. 


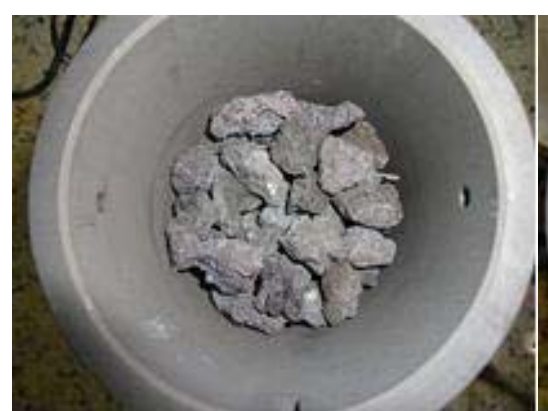

(A)

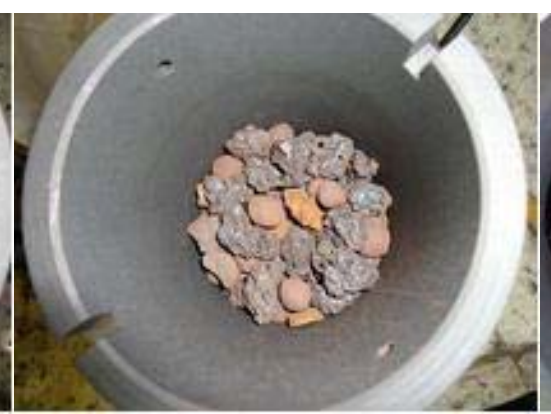

(B)

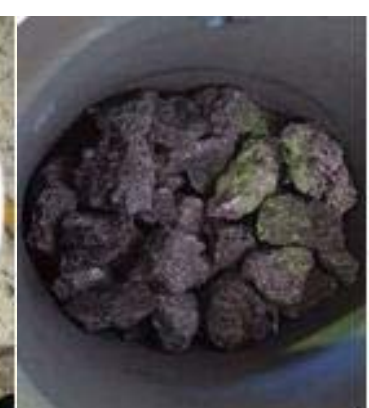

(C)

Figura 2: Carregamento do Cadinho para o ensaio de A\&F (A) primeira camada de coque (B) carga metálica (sínter, granulado e pelota) (C) última camada de coque

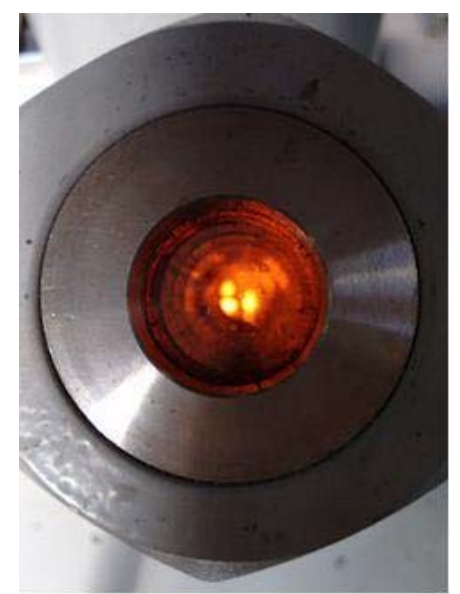

Figura 3: Momento do gotejamento de metal e escória no amostrador.

Todos os parâmetros citados na metodologia do ensaio de Amolecimento e Fusão foram medidos e/ou calculados. A Tabela 2 mostra o resumo dos principais parâmetros de cada carga metálica analisada. Cada caso será comparado com o caso Base.

Tabela 2. Resultados dos ensaios de A\&F

\begin{tabular}{|c|c|c|c|c|c|c|c|c|c|c|c|c|c|c|c|}
\hline \multirow{2}{*}{$\begin{array}{c}\text { Burden } \\
\text { (\%S/\%H/\%P) }\end{array}$} & \multirow{2}{*}{\multicolumn{2}{|c|}{ Case }} & \multirow{2}{*}{\multicolumn{2}{|c|}{$\begin{array}{cr}\text { Permeability Index } \\
\\
\mathrm{S}_{\text {area }} & \Delta \mathrm{Pmax} \\
\mathrm{Kg}{ }^{\circ} \mathrm{C} / \mathrm{cm}^{2} & \mathrm{mmH}_{2} \mathrm{O}\end{array}$}} & \multicolumn{4}{|c|}{ Temperature $\left({ }^{\circ} \mathrm{C}\right)$} & \multicolumn{4}{|c|}{ Reduction Degree (\%) } & \multicolumn{3}{|c|}{ Reduction type (\%) } \\
\hline & & & & & $\mathrm{Ts}_{200}$ & $\mathrm{Te}_{200}$ & $\Delta \mathrm{T}$ & $T_{d}$ & $\mathrm{Rs}_{200}$ & $1000^{\circ} \mathrm{C}$ & $1100^{\circ} \mathrm{C}$ & $1200^{\circ} \mathrm{C}$ & D-Red. & $\mathrm{H}_{2}$-Red & Ind-Red \\
\hline $66 / 19 / 15$ & Base & $\mathrm{S}: \mathrm{B2}=1.8 ; \mathrm{FeO}=7,0 \%$ & 17,2 & 2.959 & 1.251 & 1.381 & 129 & 1.398 & 75,6 & 39,3 & 61,5 & 73,6 & 10,4 & 13,7 & 75,9 \\
\hline $75 / 25 / 0$ & Analise I & S: Base/no Pellets & 12,7 & 2.546 & 1.253 & 1.378 & 125 & 1.396 & 76,5 & 38,9 & 62,1 & 74,4 & 11,0 & 15,0 & 74,0 \\
\hline $66 / 19 / 15$ & Analise II & S: Low B2 & 15,9 & 2.679 & 1.226 & 1.359 & 133 & 1.368 & 75,5 & 41,0 & 62,9 & 74,3 & 11,1 & 15,4 & 73,5 \\
\hline $66 / 19 / 15$ & Analise III & S: High B2 & 9,1 & 1.559 & 1.276 & 1.407 & 131 & 1.433 & 77,9 & 39,2 & 61,2 & 74,3 & 10,8 & 12,8 & 76,5 \\
\hline $66 / 19 / 15$ & Analise IV & S: High FeO & 18,0 & 3.136 & 1.238 & 1.386 & 149 & 1.397 & 72,8 & 38,3 & 60,1 & 71,2 & 14,6 & 13,0 & 72,4 \\
\hline $66 / 19 / 15$ & Analise V & S: Low FeO & 14,1 & 2.347 & 1.223 & 1.381 & 159 & 1.401 & 82,0 & 41,8 & 66,8 & 80,9 & 4,7 & 17,0 & 78,3 \\
\hline $65 / 0 / 35$ & Analise VI & S: Base/no Hematite & 16,4 & 2.704 & 1.229 & 1.387 & 159 & 1.403 & 76,8 & 40,2 & 62,8 & 75,4 & 11,0 & 15,4 & 73,7 \\
\hline
\end{tabular}

O caso Analise I, com carga mista de apenas dois componentes (sínter e granulado) apresentou posição e espessura da zona coesiva (ZC) similar à do caso Base com ligeira melhora da permeabilidade e redutibilidade.

A carga do caso Análise II, formada pelo sínter de menor Basicidade, apresentou ligeira melhora da permeabilidade, ZC com posição próxima a do caso Base (um pouco mais alta) e com espessura similar ao caso base. A redutibilidade também foi 
similar ao caso base. Podemos dizer que em relação a zona coesiva, o uso deste sínter na carga seria bem absorvido pelo processo, porém, a menor basicidade e volume de escória pioram a resistência física e a degradação sob redução do sínter, e esses não são avaliados neste ensaio pois seus efeitos são percebidos na zona granular.

A carga do caso Análise III, formada pelo sínter de maior Basicidade, foi a que apresentou melhor permeabilidade e posição da ZC em regiões mais inferiores no AF. A espessura da ZC foi semelhante ao caso base, já a redutibilidade melhorou.

A carga do caso Análise IV, com sínter de alto teor de $\mathrm{FeO}$, foi a que apresentou pior performance. A permeabilidade da ZC e a redutibilidade foram piores, quando comparado ao caso base e apresentou o maior $\Delta \mathrm{P}$ de todos os cenários analisados. A pior redutibilidade era esperada devido ao maior teor de $\mathrm{FeO}$ do Sinter.

A carga do caso Análise $\mathrm{V}$, formado pelo sinter de baixo teor de $\mathrm{FeO}$, foi a que apresentou a melhor combinação entre os índices de permeabilidade, posição e espessura da ZC e excelente redutibilidade. Essa carga, a princípio, seria a de melhor performance para o AF.

$\mathrm{O}$ caso Analise $\mathrm{VI}$, foi realizado com carga mista de apenas dois componentes (sínter e pelota), similar ao caso Analise I. A permeabilidade melhorou quando comparado ao caso Base apesar da espessura da ZC ter aumentado. A redutibilidade apresentou uma ligeira melhora.

Vale ressaltar que embora tenha ocorrido um aumento de alumina nas amostras de sínter de alto teor de $\mathrm{FeO}$ e de Basicidade baixa, não houve interferência na análise dos resultados pois, assim como o aumento do $\mathrm{FeO}$ e a redução da Basicidade, a alumina contribui negativamente com os parâmetros de amolecimento e fusão.

\section{CONCLUSÃO}

O ensaio de Amolecimento e Fusão tem sido uma boa referência para o entendimento das oscilações do perfil da Zona Coesiva do alto-forno para diferentes situações de qualidade química do sinter e de composição da carga metálica. Os resultados dos ensaios indicam coerência com o esperado pela literatura.

Foi possível concluir que o aumento da basicidade melhorou a redutibilidade e a permeabilidade da carga e o posicionamento da zona coesiva foi alterado para uma região mais baixa no alto-forno. Foi confirmado que altos teores de $\mathrm{FeO}$ no sínter é prejudicial a região de amolecimento e fusão e baixos teores de FeO melhoram a redutibilidade da carga e o desempenho do alto-forno.

Como as amostras de sinter utilizadas foram da planta industrial e as composições dos leitos de fusão são possíveis de serem praticadas no Alto-forno da CSN, esperase que os testes experimentais representem bem os resultados em escala industrial.

\section{Agradecimentos}

Agradecemos a Companhia Siderúrgica Nacional pela oportunidade de realização do trabalho e pelos recursos cedidos.

Ao Centro de Tecnologia de Ferrosos da Vale por disponibilizar suas instalações e a toda a equipe de profissionais pela realização e análise dos ensaios.

A Universidade Federal Fluminense pela participação e apoio ao desenvolvimento desse estudo.

A todos que contribuíram de forma direta ou indireta com o desenvolvimento desse trabalho. 


\section{REFERÊNCIAS}

1 Parreira A, Alencar J, Botelho M, Oliveira V, Pimenta H. Study of high temperature properties of ferrous burden formed by Pellet and Sinter. 6th International Congress on the Science and Technology of Ironmaking - ICSTI. 2012, Rio de Janeiro, RJ, Brasil.

2 Nogueira P. F. Blast Furnace Burden Softening and Melting Phenomena. PhD Thesis, Carnegie Mellon University, Pittsburgh, PA, USA, 2003.

3 Castro A, Pimenta H, Carneiro R. Zona coesiva do alto-fomo: a CVRD se capacita para abrir esta caixa preta. Revista Metalurgia \& Materiais, ABM, 2004, Vol. 60, 548, p.547549.

4 Alencar J, Parreira A, Oliveira V, Wagner D, Ricardo M. Propriedades a altas temperaturas de cargas mistas: Minério - Small Coke e Minério - HBI. 43o Seminário de Redução de Minério de Ferro e Matérias-primas. 2014, Belo Horizonte, MG, Brasil.

5 Castro A. Avaliação de Propriedades em Altas Temperaturas de Pelotas para AltoForno. Dissertação de Mestrado, UFOP, MG, Brasil, 2006. 